\title{
Heavy quark production at HERA and its relevance to the LHC
}

\author{
Matthew Wing (UCL)
}

- Introduction

- Measurements and description of heavy quark production

- Heavy quark contribution to the proton structure

- Universality of fragmentation

- Summary

DIS 2006 


\section{Introduction and motivation}




\section{Why study heavy quarks?}

To understand and probe QCD in as much detail as possible

Parton densities of proton and photon need to be precise. of future colliders, $p p, e^{+} e^{-}$and $\gamma \gamma, \ldots$

QCD-production rate should be accurately understood which can be a significant background to "new" physics

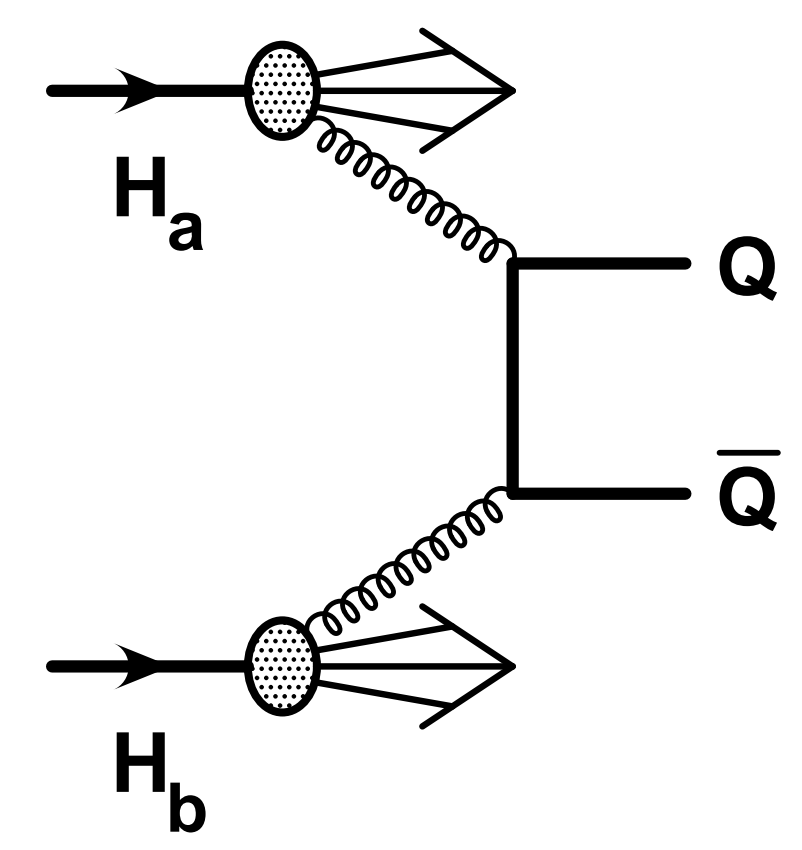




\section{Why study heavy quark production?}

Example of why the heavy quark production rate needs to be understood (ATLAS TDR) N

Consider $b \bar{b} H / A$ with $H / A \rightarrow b \bar{b}$ production at the LHC

Even requiring four $b$ jets, $S / B \sim$ few $\%$

Background from gluon splitting to $b \bar{b}$ and $g g \rightarrow b \bar{b}$

$5 \sigma$ discovery possible if QCD backgrounds known precisely

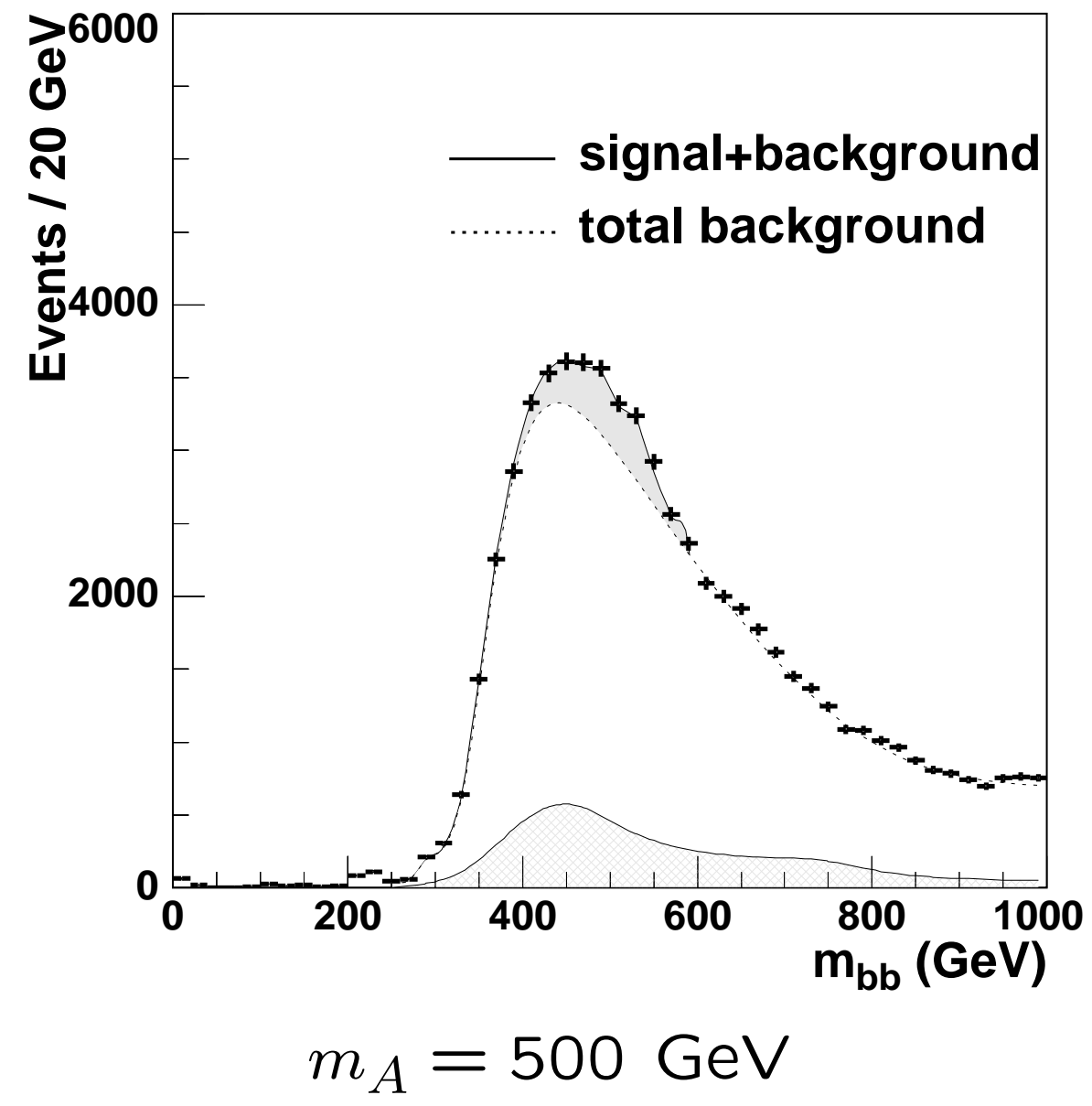

How well is the QCD background known? 


\section{Generic nature of production}

HERA

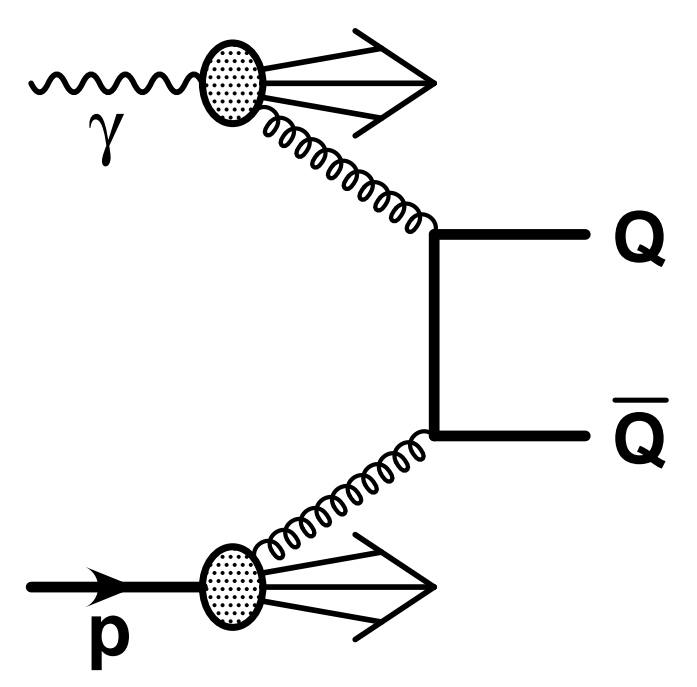

Tevatron/LHC

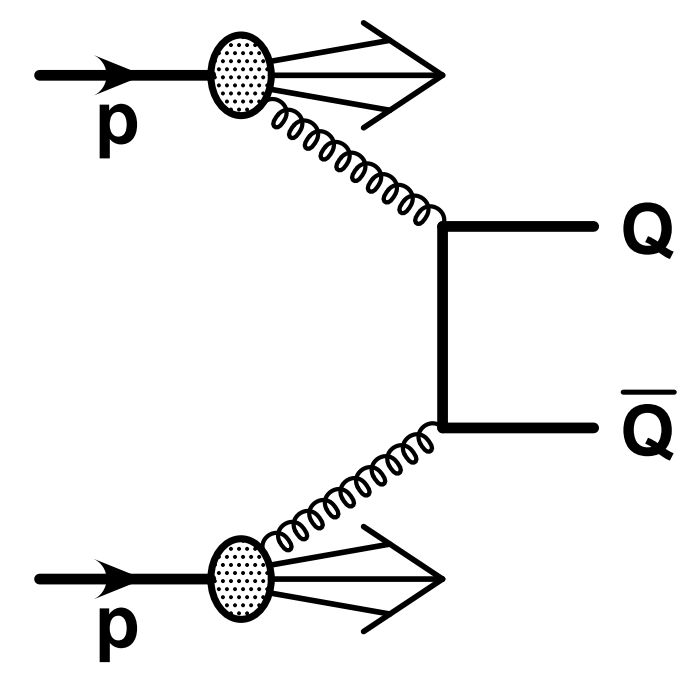

\section{PDF $\rightarrow$}

Structure functions of proton and photon

Direct sensitivity to the gluon content
Hard scatter $\rightarrow$ Dynamics of NLO QCD Implementation of diagrams and dynamics into programs

\section{Fragmentation}

Description of partonhadron transition Non-perturbative, but essential. 


\section{Theoretical calculations}

Discriminating different schemes

Fixed order:

- Frixione et al. (FMNR) used for charm and beauty in photoproduction.

- Harris and Smith (HVQDIS) used for charm and beauty in deep inelastic scattering.

Next-to-leading log:

- Cacciari et al. and Kniehl et al. used for charm and beauty in photoproduction. NB. only inclusive production, cannot do e.g. dijets

- No program for deep inelastic scattering.

Combined, FONLL (Cacciari et al.), used for charm in photoproduction.

New program, MC@NLO (Frixione and Webber), which is the fixed-order program with parton showers and hadronisation from HERWIG - not available for HERA. 


\section{Fragmentation of heavy quarks}

Reliant on non-perturbative models, e.g. Lund, cluster to describe fragmentation

NLO QCD calculations usually add on a Peterson function

Are the models and current parametrisations correct?

- Measure the fragmentation function

- Make measurements where the effects are reduced; jets, high energy 


\section{What can HERA provide and the LHC want to know?}

General state of description (by MC, NLO) of heavy quark production processes:

- where the heavy quark is produced in the hard scatter

- no direct information on gluon splitting

Information on the proton PDFs:

- gluon content information is good

- heavy quark content is for the future

Details of the fragmentation in a hadronic environment; fragmentation functions, fractions, etc..

No information yet on mulitparton interactions in heavy quark processes.

Can provide information on topology of events and jets useful for designing algorithms/triggers. 


\section{Open heavy quark production}




\section{Beauty production at the Tevatron Run I}

Significant disagreement with QCD

Extrapolation to the the $b$ quark level $\rightarrow$

Measurement of $B$ mesons gives more reliable results

Led to improvement in theory

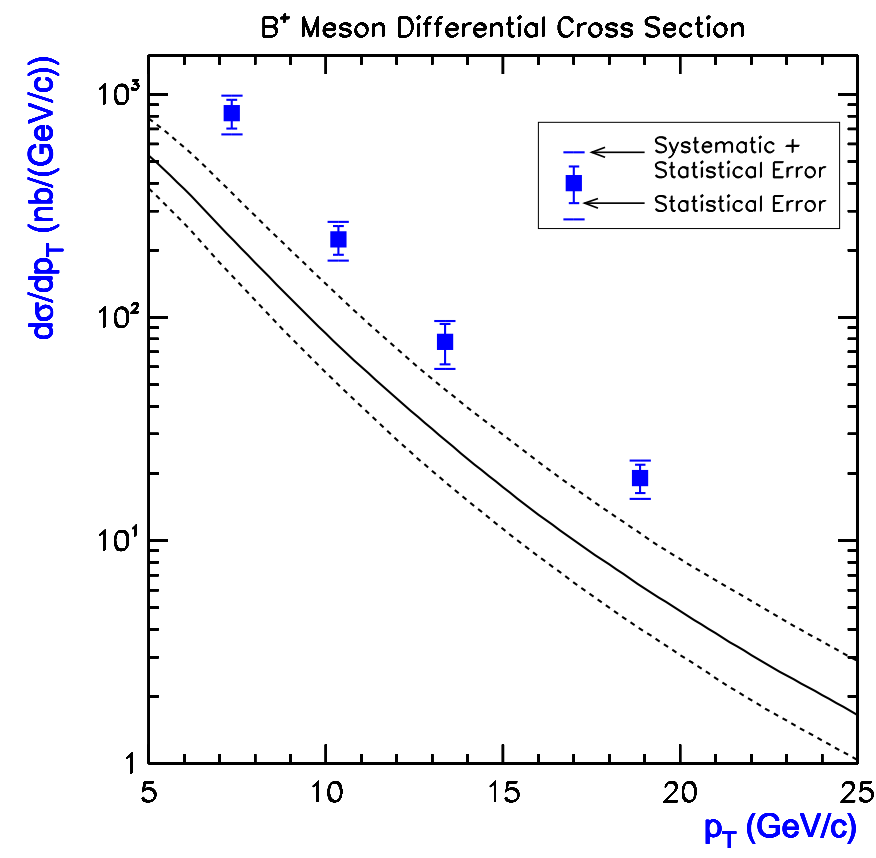

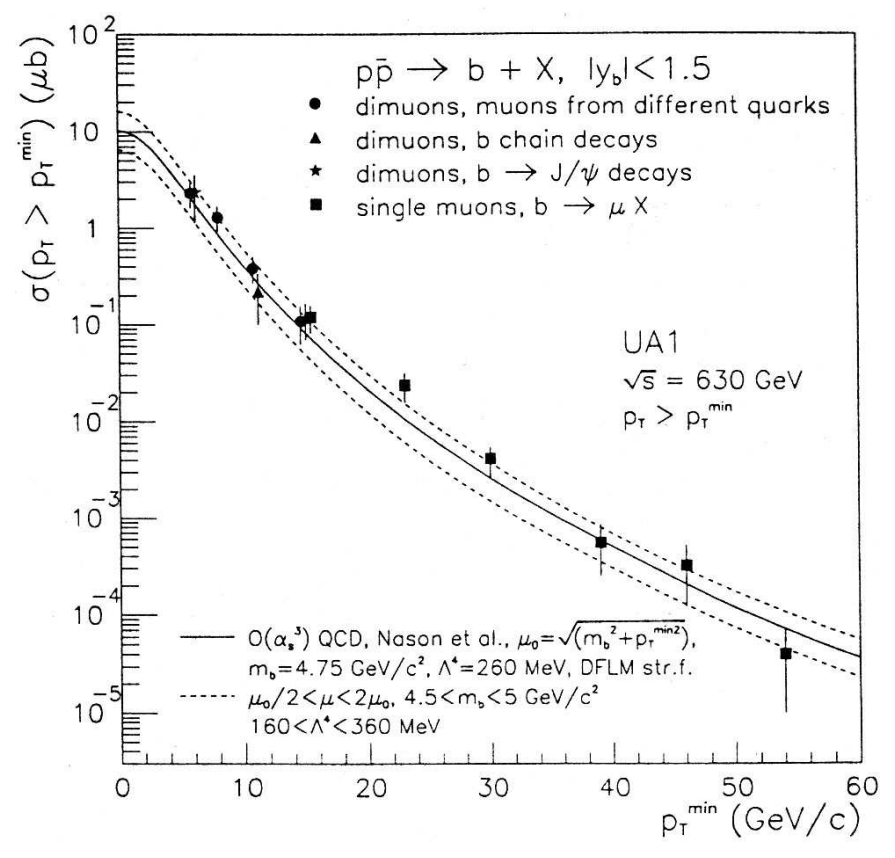
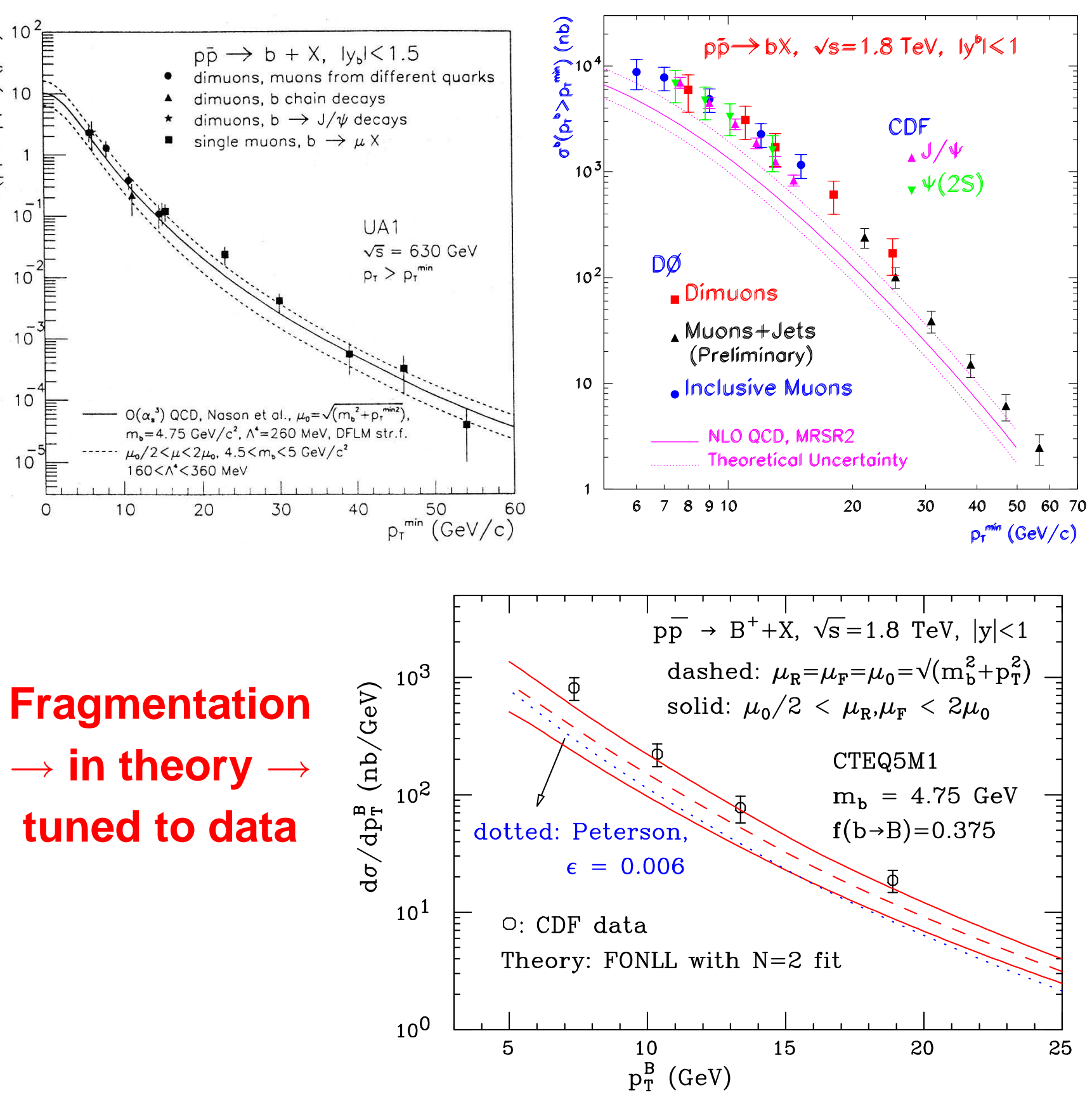


\section{Open beauty measurements at HERA}
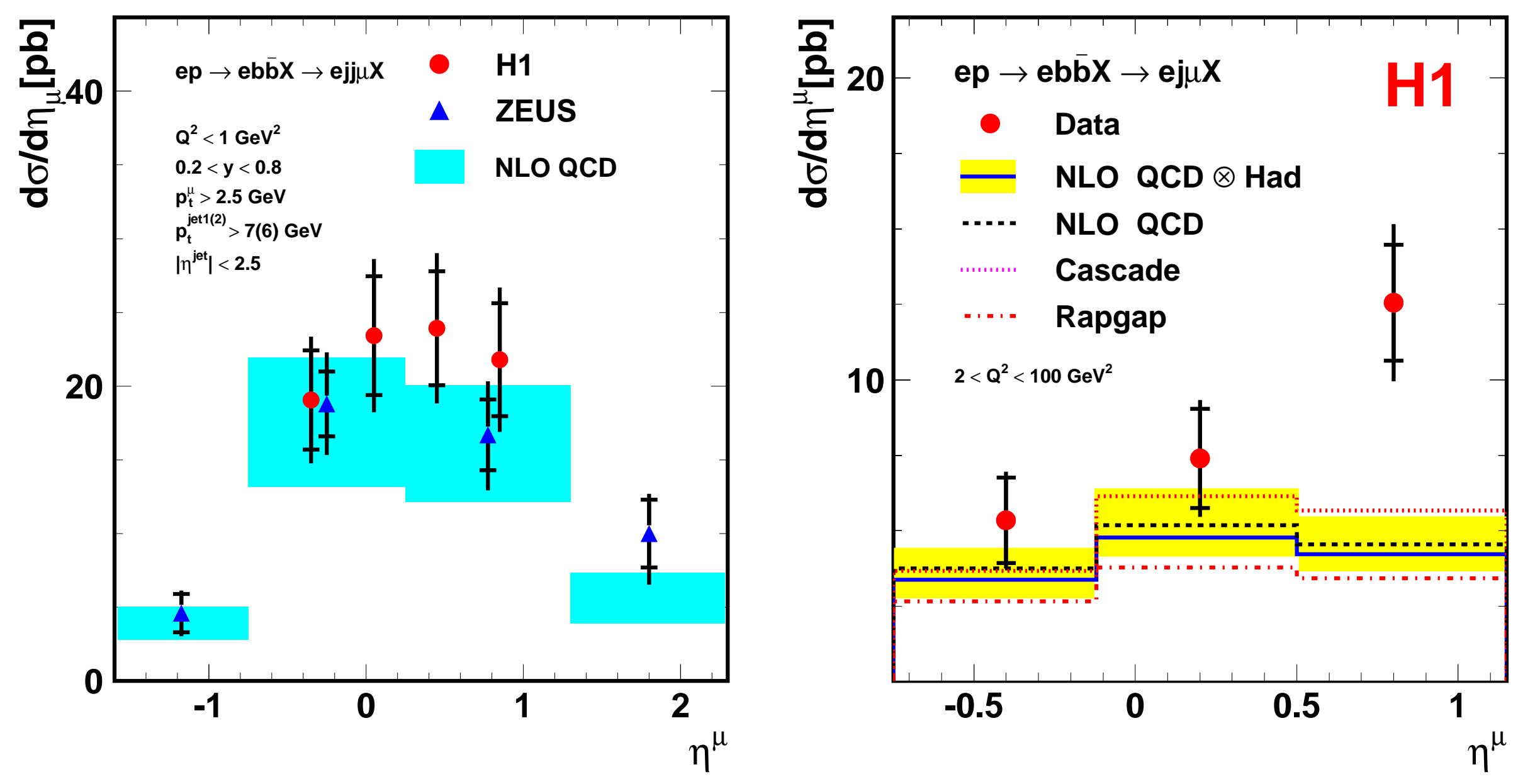

Measurements in acceptance of detector

Data from both experiments agrees okay; NLO QCD describes the data reasonably well 


\section{Latest TeVatron beauty measurement}

Run II data compared with latest theory. inclusive measurements.

Improved theory, FONLL (and MC@NLO), describe the data well.

Clearly defined measured cross section

0
$\frac{0}{0}$
0
0
0
$\frac{0}{2}$
$\frac{3}{3}$
$\frac{1}{2}$
$\frac{2}{2}$
$\frac{1}{2}$
$\frac{7}{2}$
$\frac{1}{0}$
$\frac{0}{0}$
$\frac{0}{0}$

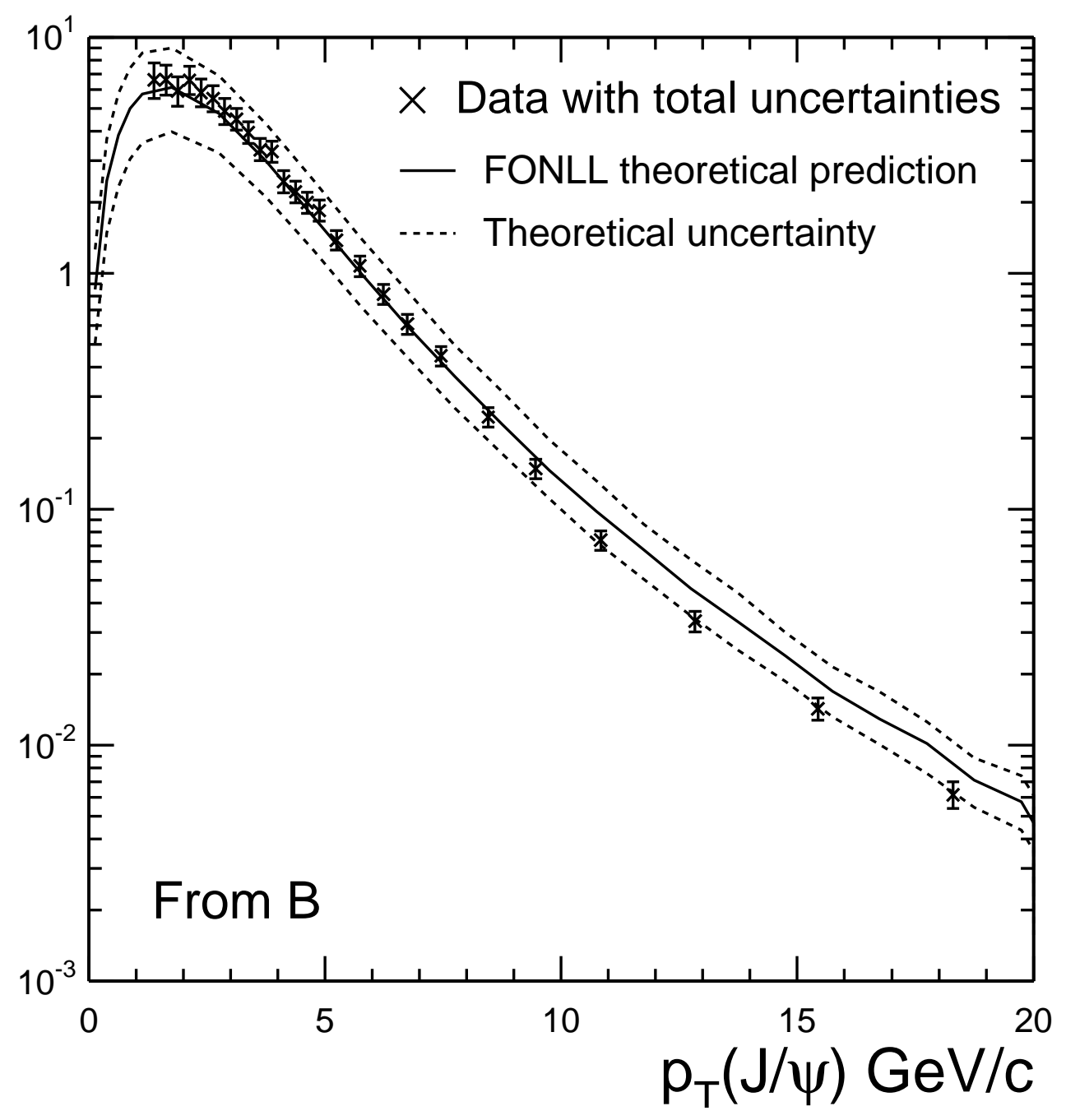

Predictions of FONLL and MC@NLO at HERA would useful. 


\section{Global comparison with hadron colliders}

\section{O.Gutsche, J. Butterworth, A. Geiser, B. Waugh}
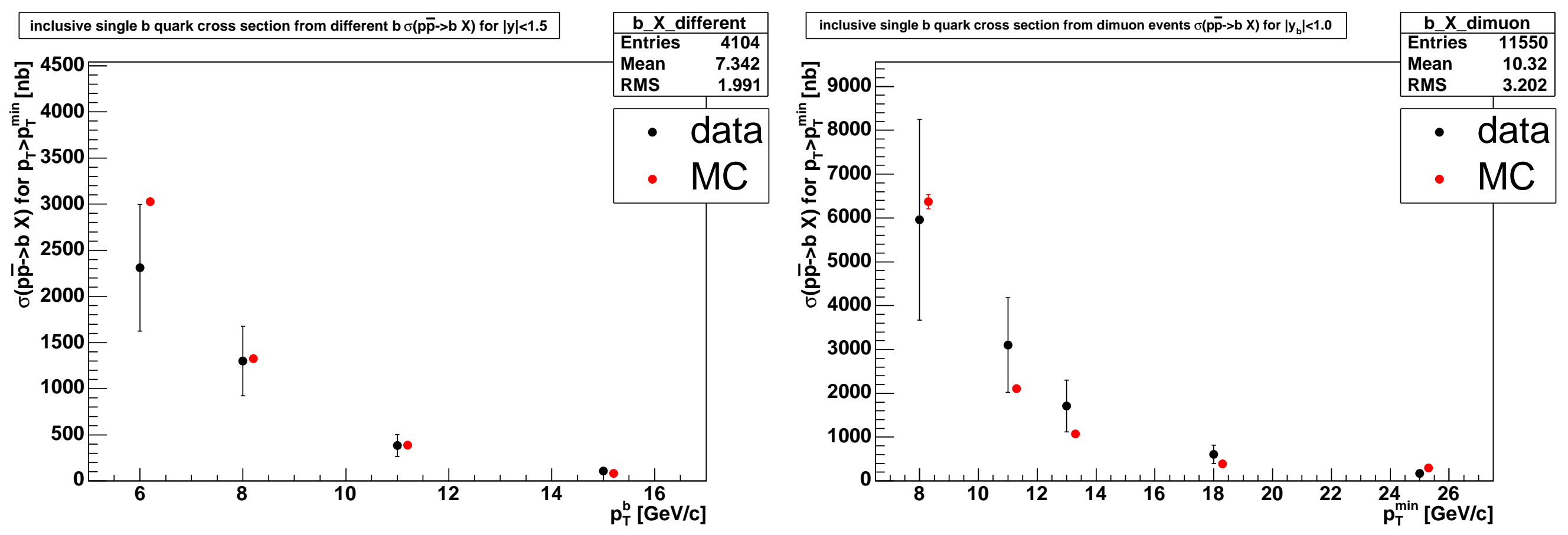

Good description of UA1 (630 GeV) and D0 (1800 GeV) data using same model.

Good MC model to use for LHC predictions: normalisation is good, complete final state.

Need to compare to all hadron-hadron data; being built up. 


\section{Comparison with HERA}
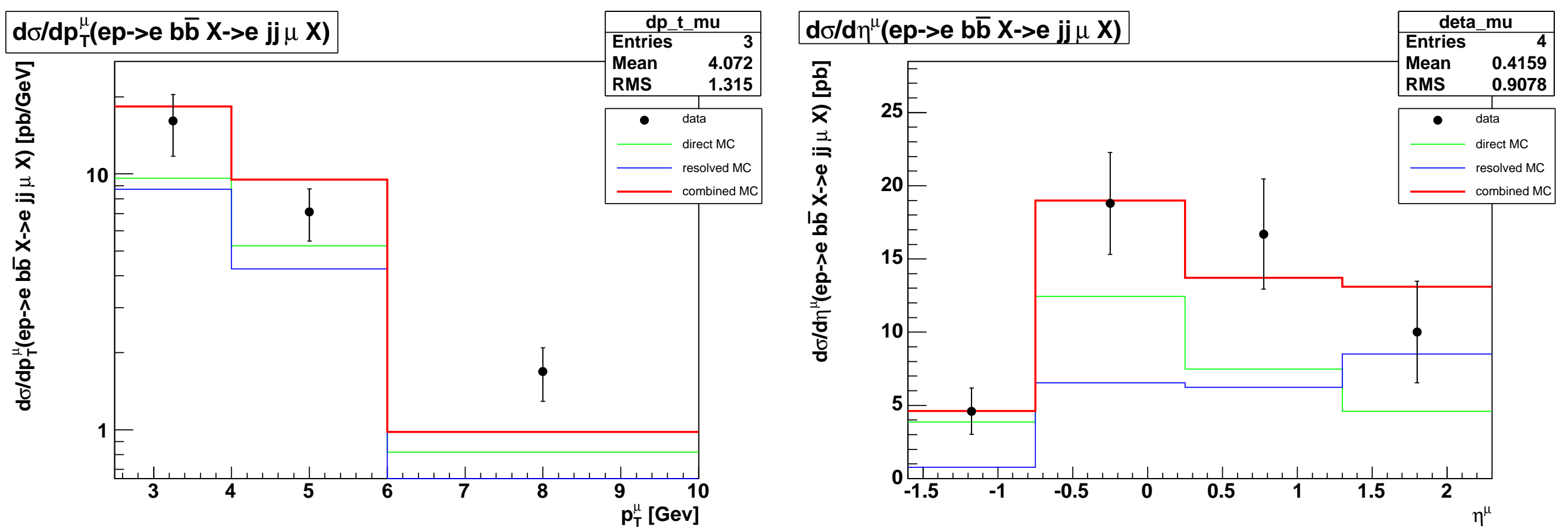

Good description of HERA data.

Shows the value of general-purpose calculations.

Have shown examples of where the procedure works. Not perfect description of all variables (yet).

But the best model so far? 


\section{Beauty discussion}

Situation developed rapidly and conclusions changed, but converging

Many different kinematic regions measured and assumptions made; treat extrapolations with caution

Data are described by NLO QCD, but issues remain

Should treat any extrapolation with caution; large extrapolation factors, into unmeasured regions, uncertainties considered...

More data is needed and is coming...

More calculations needed and improved phenomenological understanding

Global fits to all data would be useful for checking consistency

Need programs which can describe all reactions e.g MC@NLO should be able to calculate all cross sections 


\section{Charm in deep inelastic scattering}

HERA, D* in DIS

NLO (HVQDIS) prediction gives reasonable description of the data

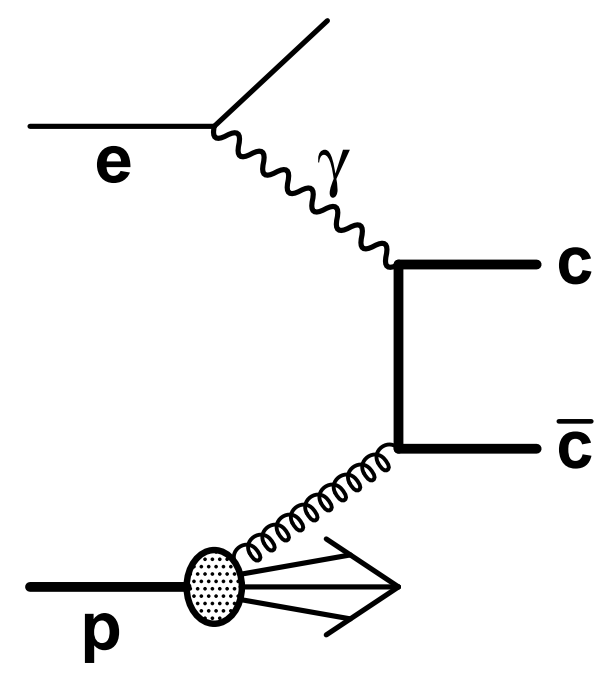

Sensitive to the gluon density in proton $\rightarrow$ more later

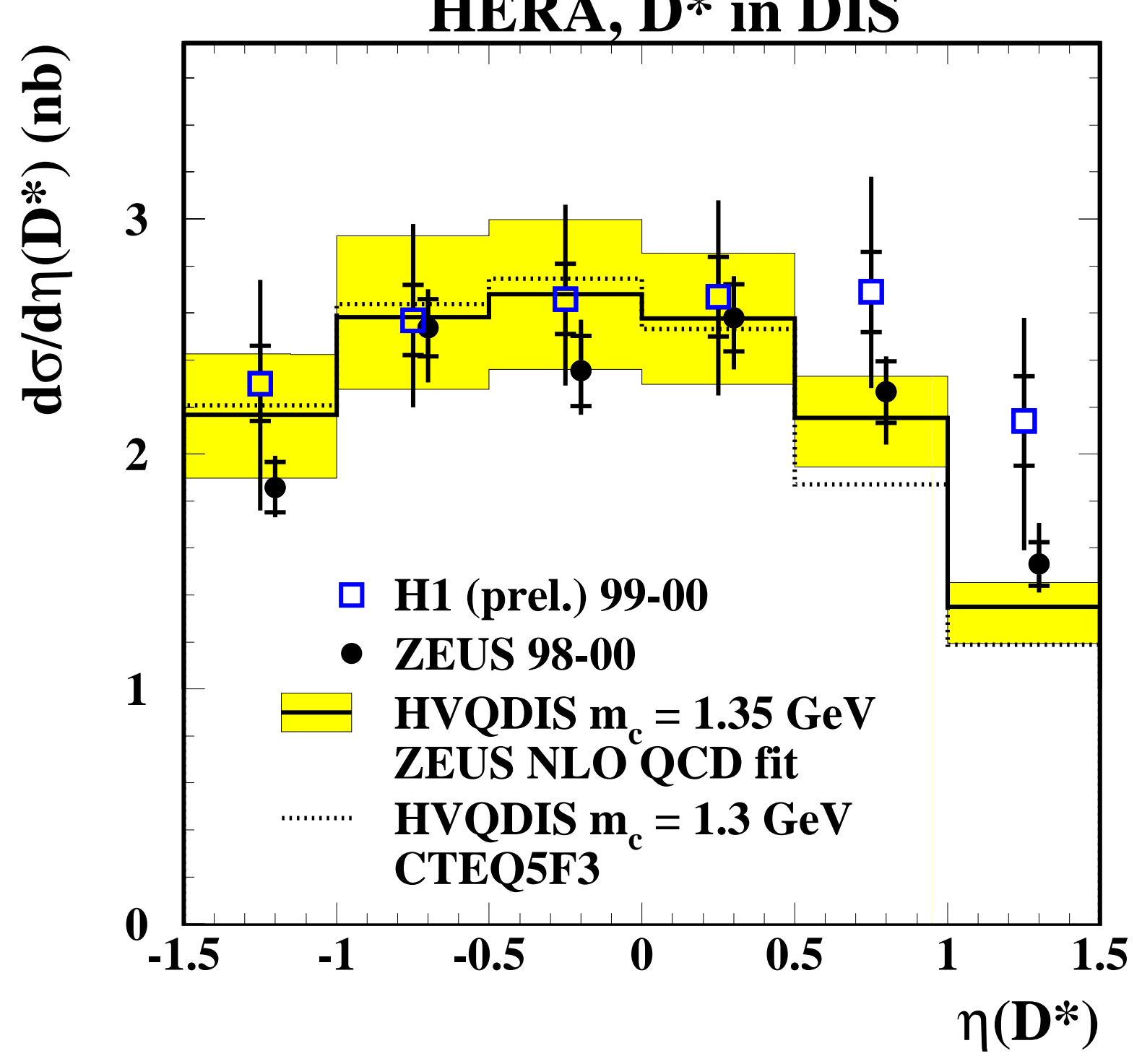




\section{Charm in photoproduction}

ZEUS
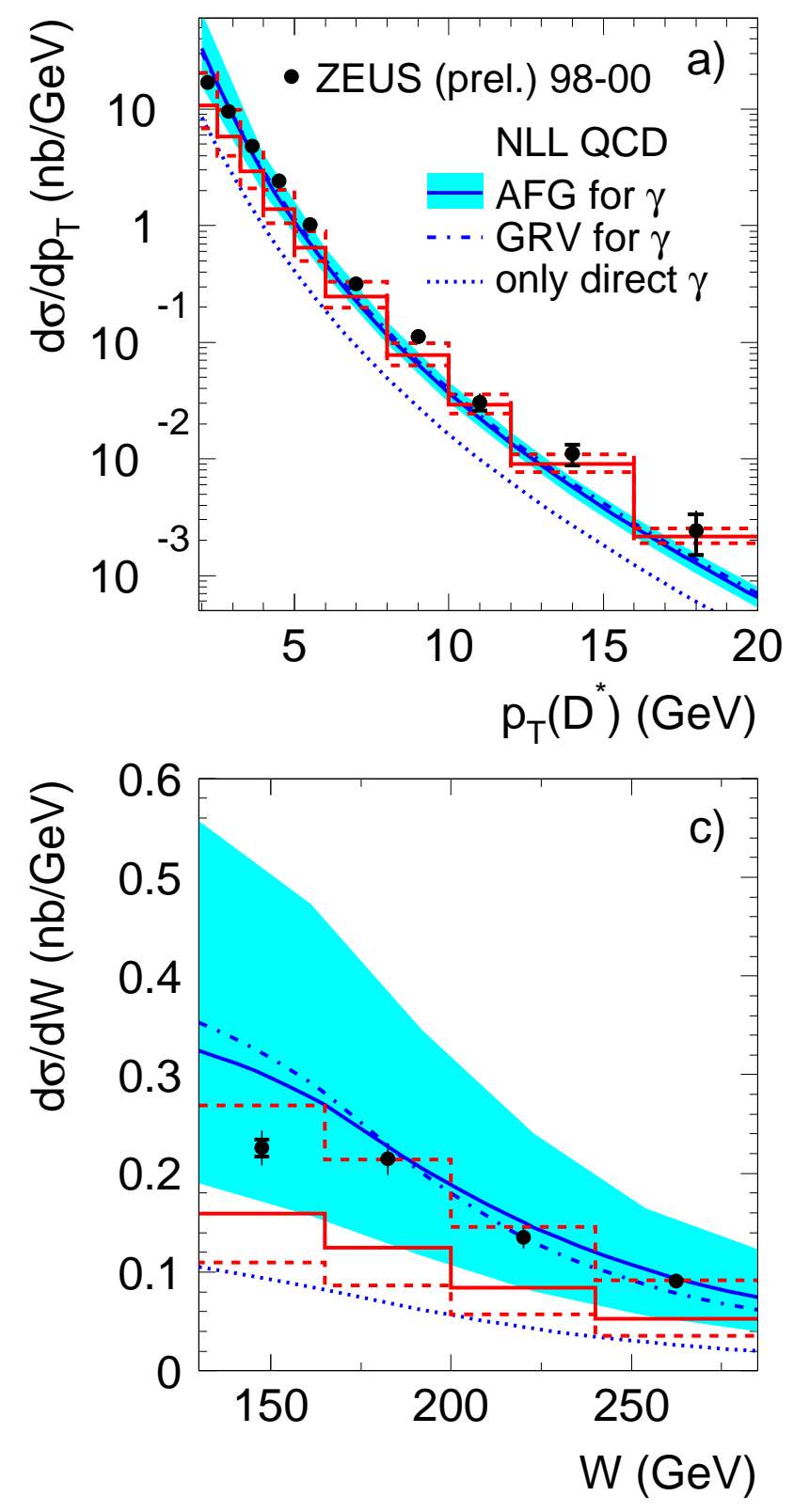
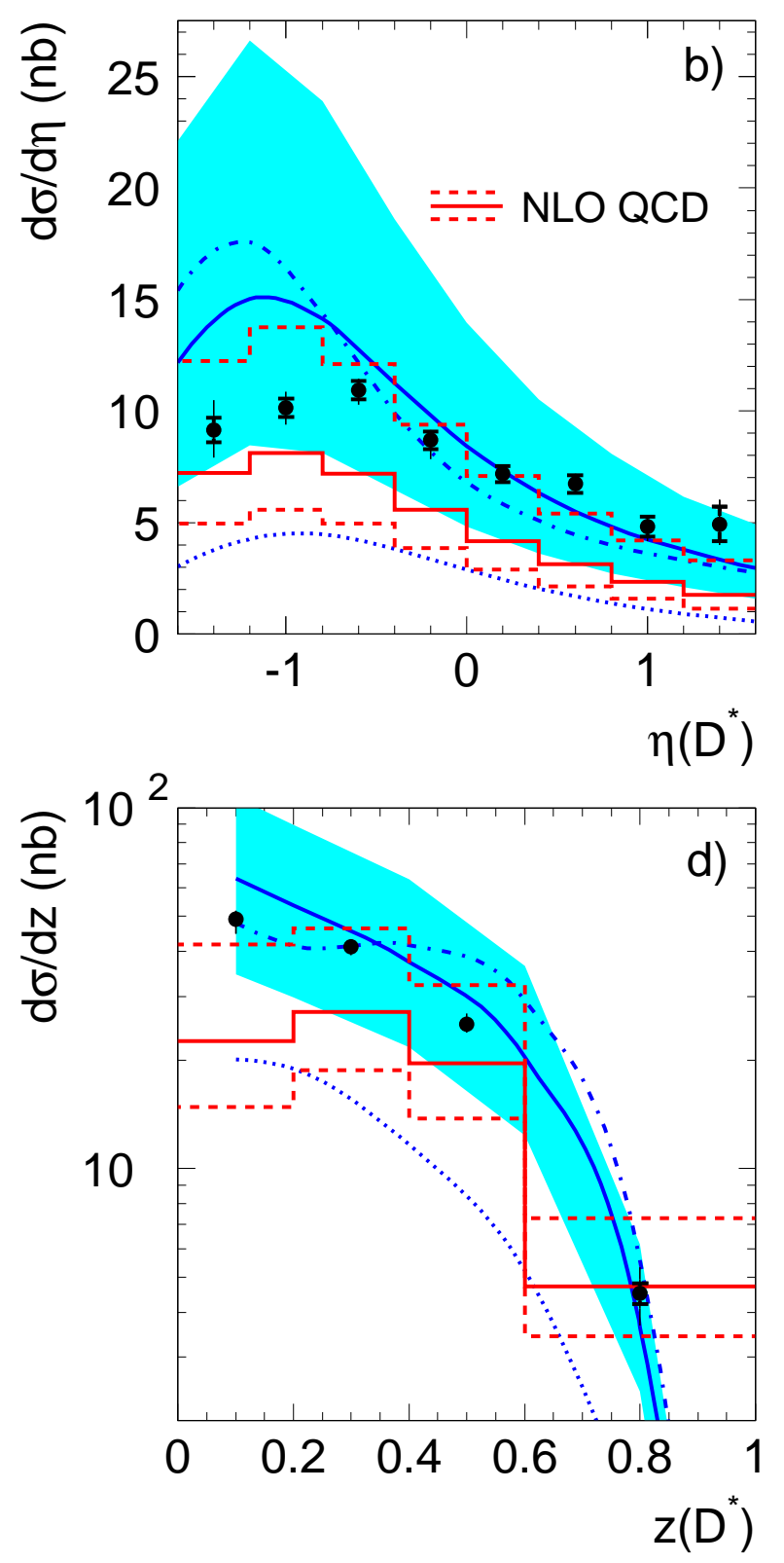

Description by NLO/NLL QCD not perfect.

General description with caveats - double differential cross sections highlight differences

Is QCD really predictive?

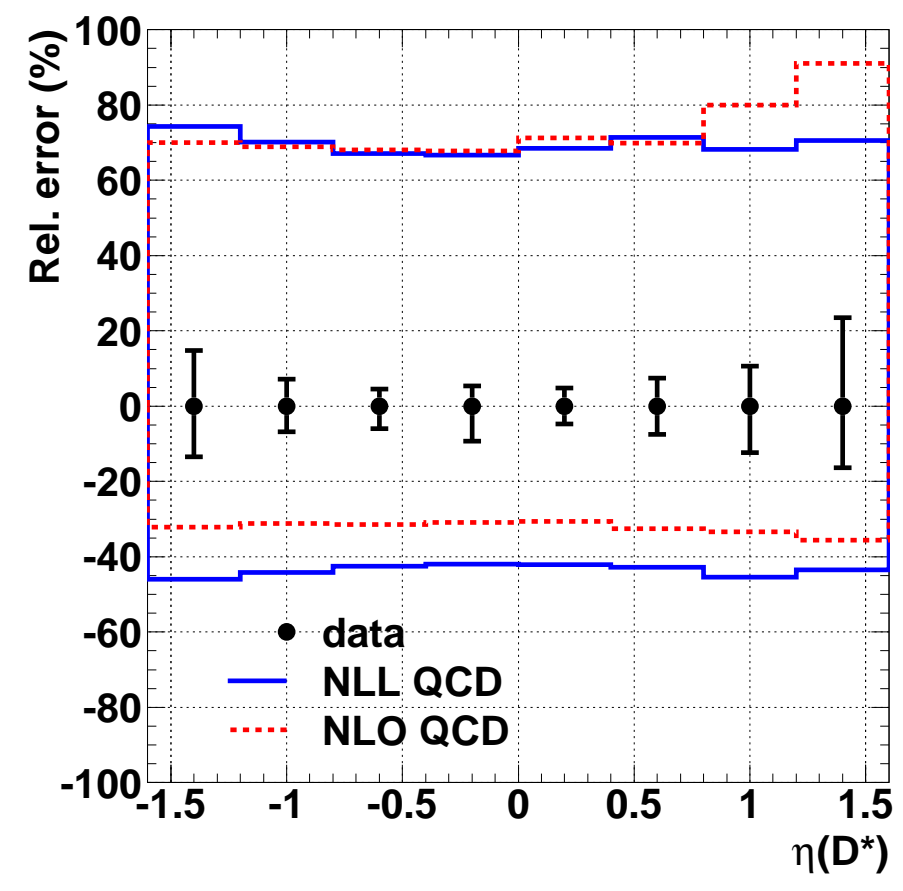

Data also good for tuning theory 


\section{Charm in dijet photoproduction}

Isolated areas where NLO

calculation does not describe data

- low $x_{\gamma}^{\text {obs }}$

- regions where higher-order contributions are large
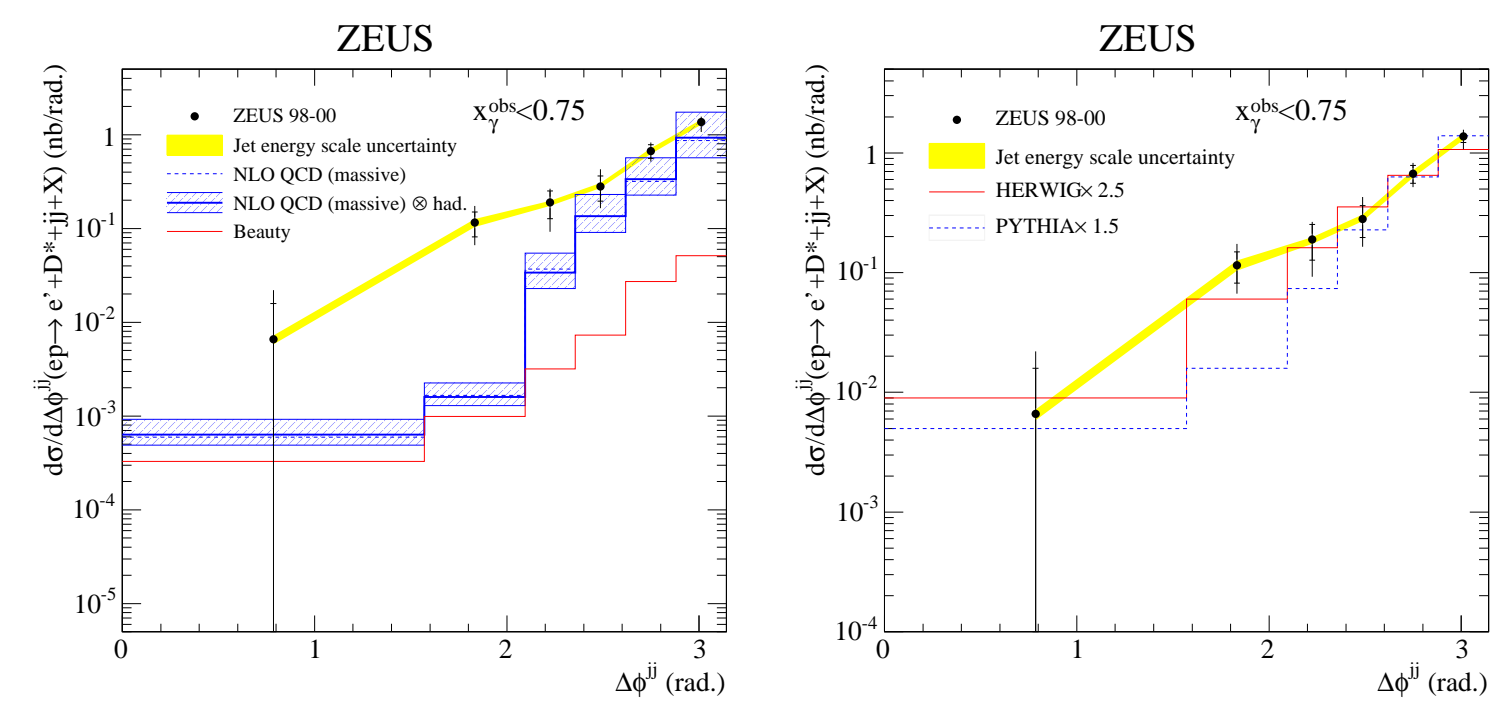

Summary

Overall description of charm photoproduction within $\sim \mathbf{5 0} \%$ and within (large) theoretical uncertainties.

But assuming your NLO calculation is right everywhere: e.g. designing a trigger; calculating QCD backgrounds, could get you into trouble. 


\section{Heavy quark contribution to the proton structure}




\section{Proton structure}

$$
\frac{d^{2} \sigma^{Q \bar{Q}}\left(x, Q^{2}\right)}{d x d Q^{2}}=\frac{2 \pi \alpha^{2}}{x Q^{4}}\left\{\left[1+(1-y)^{2}\right] F_{2}^{Q \bar{Q}}\left(x, Q^{2}\right)-y^{2} F_{L}^{Q \bar{Q}}\left(x, Q^{2}\right)\right\}
$$

Potentially large extrapolations for tagged $D$ mesons:

$$
F_{2, \text { meas }}^{Q \bar{Q}}\left(x_{i}, Q_{i}^{2}\right)=\frac{\sigma_{i, \text { meas }}\left(e p \rightarrow D^{*} X\right)}{\sigma_{i, \text { theo }}\left(e p \rightarrow D^{*} X\right)} F_{2, \text { theo }}^{Q \bar{Q}}\left(x_{i}, Q_{i}^{2}\right)
$$

Extraction of $F_{2}^{Q \bar{Q}}\left(x, Q^{2}\right)$ is dependent on the scheme.

Inclusive measurements less reliant on models and go to $p_{T} \sim 0$ 


\section{Charm contribution to the proton structure function}
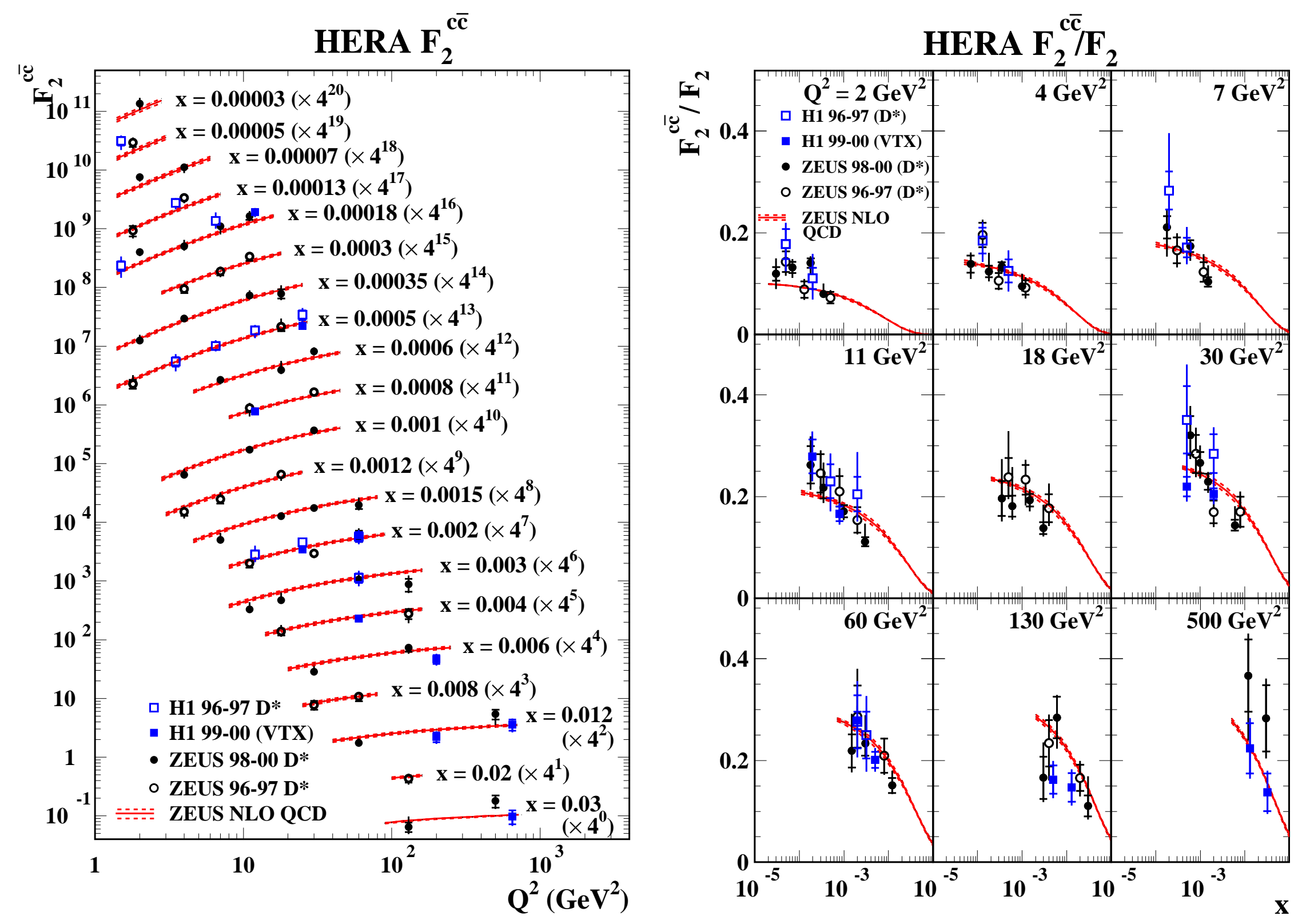

Charm production represents a significant amount of the inclusive cross section 


\section{Beauty contribution to the proton structure function}

Inclusive measurements well described by QCD

Much smaller fraction of total cross section compared to charm

Sensitivity to choice of PDF

Higher statistics and higher $Q^{2}$ will be useful for LHC

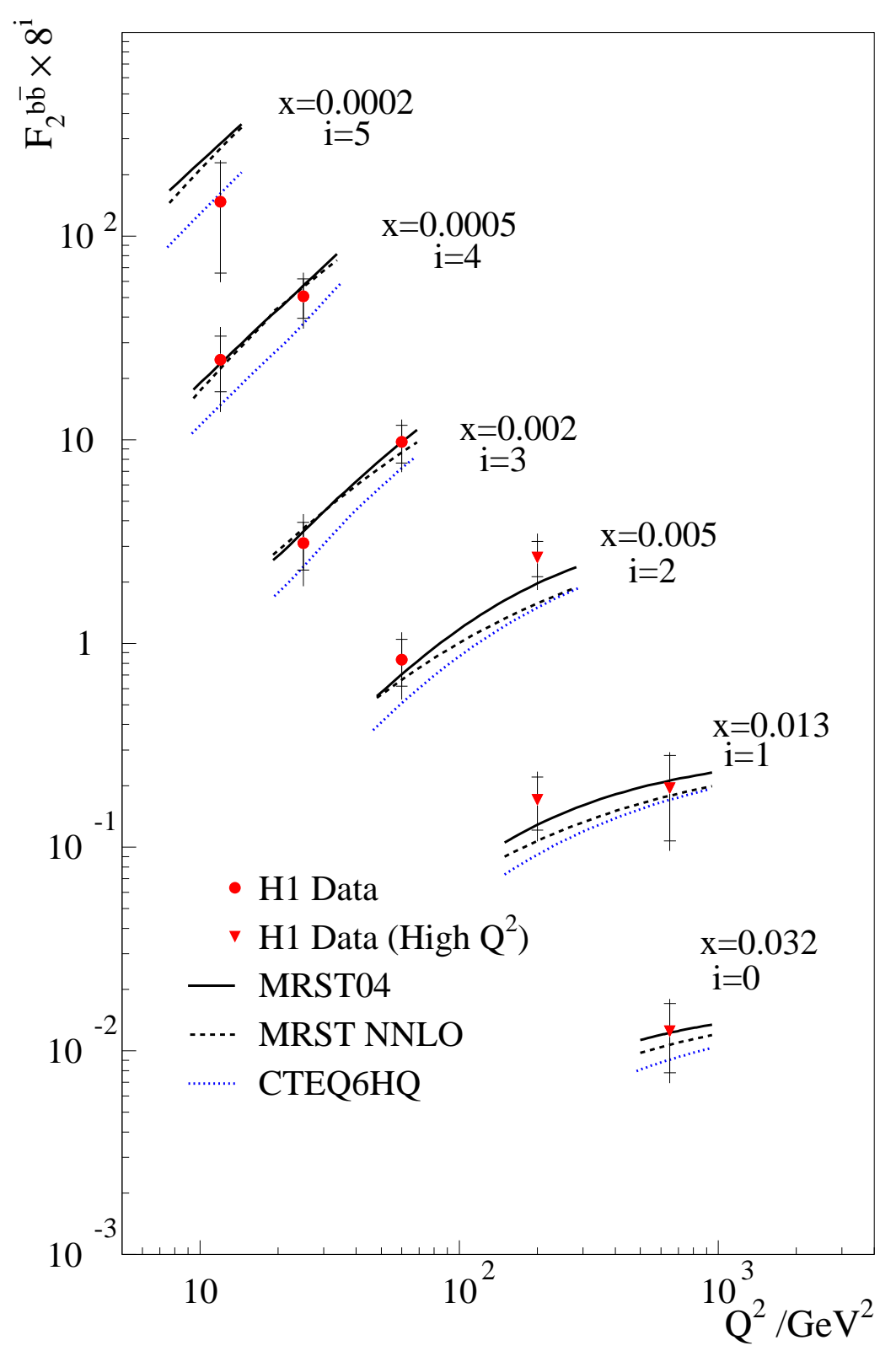




\section{Universality of fragmentation}




\section{Charm fragmentation parameters}

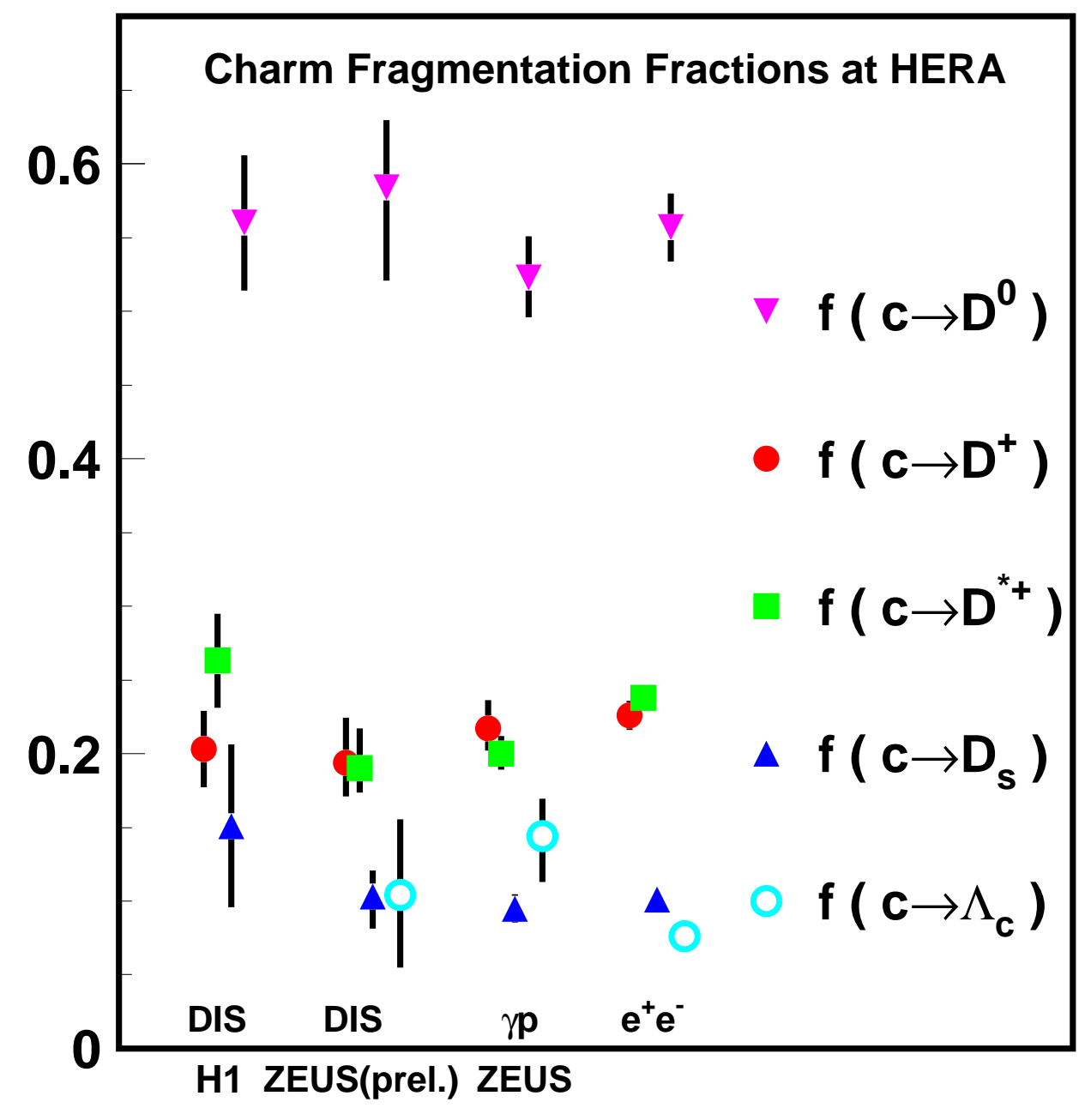

Charm fragmentation fractions are universal

Same measurements at the TeVatron could be made

Correct values being used in NLO calculations 


\section{Charm fragmentation function}

Measurements at hadronic colliders are important:

- Independent ways of measuring fragmentation

- Extra constraints and precision on fragmentation function

- Consistency in more complicated

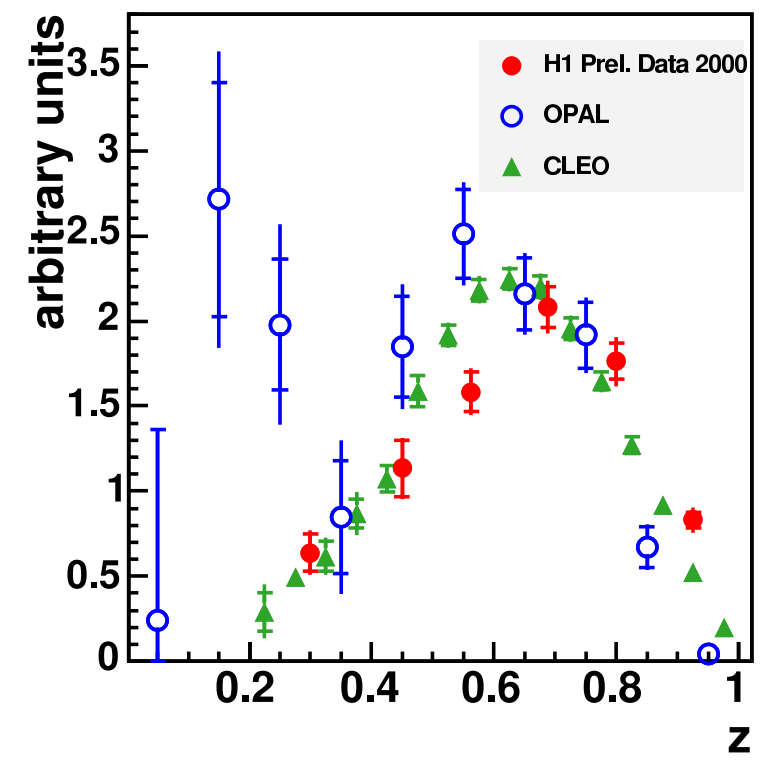

H1 hemisphere method

$<\sqrt{\mathrm{s}}>\approx 10 \mathrm{GeV}$,

$\mathrm{z}=\frac{\left(\mathrm{E}+\mathrm{p}_{\mathrm{L}}\right)_{\mathrm{D}}}{\sum_{\text {hem }}(\mathrm{E}+\mathrm{p})}$

OPAL $\sqrt{\mathrm{s}}=91.2 \mathrm{GeV}$, $\mathrm{z}=2 \mathrm{E}_{\mathrm{D}^{*}} / \sqrt{\mathrm{s}}$

CLEO $\sqrt{\mathrm{s}} \approx 10 \mathrm{GeV}$, $\mathrm{z}=\mathrm{p}_{\mathrm{D}^{*}} / \mathrm{p}_{\max }$ environment - hadron remnant?

General trend is similar for $e^{+} e^{-}$and $e p$

Different processes, energies, $z$ definitions $\rightarrow$ need consistent fit to all data in MC and NLO.

Improve measurements at HERA and hopefully have measurements at the TeVatron $\rightarrow$ useful input for the LHC. 


\section{Conclusions}

Increasing number of high precision measurements of heavy quark production from HERA.

Primary goal: measure $F_{2}^{\mathrm{QQ}}$ as accurately as possible and over as wide a kinematic range as possible.

More quantitative statement on $b$ production rates: data vs. theory

Calculations which are proven against many different processes and cross sections

Demonstration of fragmentation function universality (or not) 